\title{
Complex Manifolds
}

\author{
Research Article
}

Indranil Biswas*, Mahan Mj, and Misha Verbitsky

\section{Stable Higgs bundles over positive principal elliptic fibrations}

https://doi.org/10.1515/coma-2018-0012

Received June 17, 2018; accepted October 7, 2018.

\begin{abstract}
Let $M$ be a compact complex manifold of dimension at least three and $\Pi: M \longrightarrow X$ a positive principal elliptic fibration, where $X$ is a compact Kähler orbifold. Fix a preferred Hermitian metric on $M$. In [14], the third author proved that every stable vector bundle on $M$ is of the form $L \otimes \Pi^{*} B_{0}$, where $B_{0}$ is a stable vector bundle on $X$, and $L$ is a holomorphic line bundle on $M$. Here we prove that every stable Higgs bundle on $M$ is of the form $\left(L \otimes \Pi^{*} B_{0}, \Pi^{*} \Phi_{X}\right)$, where $\left(B_{0}, \Phi_{X}\right)$ is a stable Higgs bundle on $X$ and $L$ is a holomorphic line bundle on $M$.
\end{abstract}

Keywords: Principal elliptic fibration, Higgs bundle, Preferred metric, Yang-Mills equation, Positivity

MSC: 14P25, 57M05, 14F35, 20F65, 57M50, 57M07, 20F67

\section{Introduction}

Stable holomorphic vector bundles on compact Kähler manifolds and, more generally, on compact Hermitian manifolds are extensively studied. On of the basic results in this topic is that an indecomposable holomorphic vector bundle $E$ on a compact complex manifold $X$ equipped with a Gauduchon metric $g_{X}$ admits a Hermitian structure $h_{E}$ that solves the Hermitian-Einstein equation if and only if $E$ is stable [2, 7, 8, 13].

A principal elliptic fibration is a holomorphic principal bundle with an elliptic curve as the structure group. Let $\left(X, \omega_{X}\right)$ be a compact Kähler orbifold. A principal elliptic fibration $\Pi: M \longrightarrow X$ is called positive if the differential form $\Pi^{*} \omega_{X}$ is exact. For a positive principal elliptic fibration $(M, \Pi)$, the complex manifold $M$ is never Kähler. There is a notion of preferred Hermitian metric on the total space $M$ of a positive principal elliptic fibration $(M, \Pi)$ which depends on the Kähler form $\omega_{X}$ defined on the base $X$ of the fibration (the definition of a preferred Hermitian metric is recalled in Section 2.1).

Let $(M, \Pi)$ be a positive principal elliptic fibration equipped with a preferred Hermitian metric, such that $\operatorname{dim}_{\mathbb{C}} M \geq 3$. In [14], the third author investigated the stable vector bundles on $M$. The main result of [14] says that every stable vector bundle $E$ on $X$ is of the form $L \otimes \Pi^{*} B_{0}$, where $L$ is a line bundle on $M$ and $B_{0}$ is a stable vector bundle on $\left(X, \omega_{X}\right)$.

\footnotetext{
*Corresponding Author: Indranil Biswas: School of Mathematics, Tata Institute of fundamental research, Homi Bhabha road, Mumbai 400005, India, and Mathematics Department, EISTI-University Paris-Seine, Avenue du parc, 95000, Cergy-Pontoise, France, E-mail: indranil@math.tifr.res.in

Mahan Mj: School of Mathematics, Tata Institute of Fundamental Research, Homi Bhabha Road, Mumbai 400005, India, E-mail: mahan@math.tifr.res.in

Misha Verbitsky: Instituto Nacional de Matemática Pura e Aplicada (IMPA) Estrada Dona Castorina, 110, Jardim Botânico, CEP 22460-320, Rio de Janeiro, RJ, Brasil, and Laboratory of Algebraic Geometry, Faculty of Mathematics, National Research University HSE, 6 Usacheva street, Moscow, Russia, E-mail: verbit@verbit.ru
} 
A very important topic in the study of holomorphic vector bundles is the Higgs bundles. An indecomposable Higgs bundle $(E, \theta)$ is stable if and only if the vector bundle $E$ admits a Hermitian structure that solves the Yang-Mills equation associated to $(E, \theta)[9,11]$.

Our aim here is to extend the result of [14] mentioned above to the context of Higgs bundles. Note that for a stable Higgs bundle $(E, \theta)$, the underlying vector bundle $E$ need not be stable. The techniques of [14] nevertheless can be adapted to our situation (as we do in Section 3, see Theorem 3.2) to prove the analogous statement for the holomorphic vector bundle underlying the Higgs field. It turns out that more is true: the Higgs field also is a pullback. The following is the main theorem of our paper (see Theorem 4.2):

Theorem 1.1. Let $\Pi: M \longrightarrow X$ be a positive principal elliptic fibration with $n=\operatorname{dim} M \geq 3$. Equip $M$ with a preferred Hermitian metric. Let $(E, \Phi)$ be a stable Higgs bundle on $M$. Then there is stable Higgs bundle $\left(B_{0}, \Phi_{X}\right)$ on $X$ and a holomorphic line bundle $L$ over $M$ such that the Higgs bundle $\left(L \otimes \Pi^{*} B_{0}, \Pi^{*} \Phi_{X}\right)$ on $M$ is isomorphic to $(E, \Phi)$.

In [1], the first two authors had proved an analog of Theorem 1.1 for Sasakian manifolds where the fibers are real one dimensional instead of complex one-dimensional as in the situation here. The present paper thus gives a natural context where the ideas of [14] and [1] come together.

\section{Preliminaries}

\subsection{Positive principal elliptic fibrations}

Let $T$ be a complex smooth elliptic curve with a point $0 \in T$ which is the identity element for the group structure on $T$. Let $\left(X, \omega_{X}\right)$ be a compact Kähler orbifold with $\operatorname{dim}_{\mathbb{C}} X \geq 2$; the Kähler form on $X$ is denoted by $\omega_{X}$. Take a pair $(M, \Pi)$, where

- $\quad M$ is a compact complex manifold equipped with a holomorphic action of $T$ on the right of it, and

$-$

$$
\Pi: M \longrightarrow X
$$

is a holomorphic surjective submersion that commutes with the trivial action of $T$ on $X$; moreover, the projection $\Pi$ and the action of $G$ on $M$ together make $M$ a holomorphic principal $T$-bundle over the orbifold $X$.

Triples $(X, M, \Pi)$ of the above type are called principal $T$-fibrations, and when $T$ is not fixed beforehand, quadruples $(T, X, M, \Pi)$ of the above type are called principal elliptic fibrations; see [14, p. 251].

Note that the action of $T$ on $M$ is free only when the orbifold structure of $X$ trivial (meaning $X$ is a Kähler manifold). If $X$ is a nontrivial orbifold, then the action of $T$ on $M$ can have finite isotropies on a closed analytic subspace of $M$.

We assume that the fibration $\Pi$ in (1) is positive, meaning the $(1,1)$-form $\Pi^{*} \omega_{X}$ on $M$ is exact (see [14, p. 253, Definition 2.1]).

It is known that if the total space of an principal elliptic fibration over a compact Kähler manifold is Kähler, then the principal elliptic fibration is in fact isotrivial (pullback to some finite cover of base is a trivial fibration). On the other hand, given a trivial fibration and a differential form $\alpha$ on the base, if the pullback of $\alpha$ to the total space is exact, then $\alpha$ is exact. Therefore, the positivity of $\Pi$ implies that $M$ is not a Kähler manifold.

We note that an important class of positive principal elliptic fibrations is quasi-regular Vaisman manifolds [14, p. 253, Example 2.2], [3, 10].

Take a positive principal $T$-fibration $(X, M, \Pi)$. A Hermitian metric $g$ on $M$ is called preferred if

- $\quad g$ is preserved by the action of $T$ on $M$, and 
- $\quad$ the map $\Pi$ from $(M, g)$ to $\left(X, \omega_{X}\right)$ is a Riemannian submersion, meaning the restriction of the differential $d \Pi: T M \longrightarrow T X$ of $\Pi$ to the orthogonal complement kernel $(d \Pi)^{\perp} \subset T M$ is an isometry.

(See [14, p. 254, Definition 2.6].)

A Hermitian metric on $g_{Z}$ on a complex manifold $Z$ of dimension $\delta$ is called Gauduchon if the corresponding (1,1)-form $\omega_{Z}$ satisfies the equation [4]:

$$
\partial \bar{\partial} \omega_{Z}^{\delta-1}=0
$$

It is known that a preferred metric on a positive principal T-fibration is Gauduchon [14, p. 257, Proposition 4.1].

\subsection{Yang-Mills connections}

Let $Y$ be a compact complex manifold of dimension $\delta$. A Higgs field on a holomorphic vector bundle $V$ over $Y$ is a holomorphic section

$$
\Phi \in H^{0}\left(Y, \operatorname{End}(V) \otimes \Omega_{Y}^{1}\right)
$$

such that the section $\Phi \wedge \Phi$ of $\operatorname{End}(V) \otimes \Omega_{Y}^{2}$ vanishes identically [11, 12]. A Higgs bundle on $Y$ is a holomorphic vector bundle on $Y$ equipped with a Higgs field.

Fix a Gauduchon metric $g_{Y}$ on $Y$; let $\omega_{Y}$ be the corresponding $(1,1)$-form. The degree of a sheaf on $Y$ will be defined using $\omega_{Y}$. For a torsion-free coherent analytic sheaf $F$ on $Y$, the real number $\operatorname{degree}(F) / \operatorname{rank}(F)$, which is called the slope of $F$, is denoted by $\mu(F)$.

A Higgs bundle $(V, \Phi)$ is called stable (respectively, semistable) if for every coherent analytic subsheaf $F \nsubseteq V$ such that $V / F$ is torsion-free, and $\Phi(F) \subset F \otimes \Omega_{Y}^{1}$, the inequality

$$
\mu(F)<\mu(V) \text { (respectively, } \mu(F) \leq \mu(V) \text { ) }
$$

holds. A Higgs bundle $(V, \Phi)$ is called polystable if it is semistable and isomorphic to a direct sum of stable Higgs bundles.

Let $V$ be a Hermitian holomorphic vector bundle over $Y$. The Chern connection on $V$ will be denoted as $\nabla$. Let

$$
\Theta \in C^{\infty}\left(Y ; \operatorname{End}(V) \otimes \Omega_{Y}^{1,1}\right)
$$

be the curvature of $\nabla$. Define the operator

$$
\Lambda: \operatorname{End}(V) \otimes \Omega_{Y}^{1,1} \longrightarrow \operatorname{End}(V)
$$

to be the Hermitian adjoint of the multiplication operator $b \longmapsto b \otimes \omega$. Let $\Phi$ be a Higgs field on $V$. The connection $\nabla$ is called Yang-Mills if

$$
\Lambda\left(\Theta+\left[\Phi, \Phi^{*}\right]\right)=c \cdot \operatorname{Id}_{V}
$$

for some $c \in \mathbb{C}[11,12]$.

It is known that given a Higgs bundle $(V, \Phi)$, the vector bundle $V$ admits a Hermitian structure that satisfies the Yang-Mills equation for $(V, \Phi)$, if and only if $(V, \Phi)$ is polystable $[8,9,11]$.

\section{Vector bundles underlying stable Higgs bundles are pullbacks}

As before, $\left(X, \omega_{X}\right)$ is a compact Kähler orbifold, and $T$ is an elliptic curve with a base point. Let $M$ be a compact complex manifold of dimension $n$, with $n \geq 3$. Let

$$
\Pi: M \longrightarrow X
$$


be a positive principal $T$-fibration. Let

$$
T_{v} \subset T M
$$

be the line subbundle given by the action of $T$ on $M$. So $T_{v}$ is the vertical tangent bundle for the projection $\Pi$.

Fix a preferred Hermitian metric $g$ on $M$. Let $\omega$ be the $(1,1)$-form on $M$ associated to $g$. This form $\omega$ is not closed, because $g$ is not Kähler [6].

The orthogonal complement $T_{v}^{\perp}$ will be denoted by $T_{h}$. We have an orthogonal decomposition

$$
\omega=\omega_{v}+\omega_{h},
$$

where $\omega_{v}$ vanishes on $T_{h}$ and $\omega_{h}$ vanishes on $T_{v}$.

For a point $y \in M$, choose an orthonormal basis $\theta_{0}, \theta_{1}, \cdots, \theta_{n-1}$ of $\left(T_{y}^{1,0} M\right)^{*}$, such that $\theta_{0} \in \omega_{v}(y)$ and $\theta_{j} \in \omega_{h}(y)$ for $1 \leq j \geq n-1$. Then as in [14],

- $\omega=\sqrt{-1} \sum_{j \geq 0} \theta_{j} \wedge \overline{\theta_{j}}$, and

$-\Pi^{*} \omega_{X}=\sqrt{-1} \sum_{j \geq 1} \theta_{j} \wedge \overline{\theta_{j}}$.

Let $E$ be a Hermitian holomorphic vector bundle on $M$. Let $\nabla$ be the Chern connection on $E$; the curvature of $\nabla$ will be denoted by $\Theta$. Note that $\Theta \in C^{\infty}\left(M, \Omega_{M}^{1,1} \otimes \operatorname{End}(E)\right)$ such that $\nabla(\Theta)=0$ (Bianchi identity), and $\Theta^{*}=\Theta$; we recall that $(A \otimes \alpha)^{*}=A^{*} \otimes \bar{\alpha}$ for any $A \in \operatorname{End}\left(E_{y}\right)$ and $\alpha \in \Omega^{p, q}(M)_{y}$. Therefore, $\Theta(y)$ uniquely decomposes as

$$
\Theta(y)=\sum_{i \neq j}\left(\theta_{i} \wedge \overline{\theta_{j}}+\theta_{j} \wedge \overline{\theta_{i}}\right) \otimes b_{i j}+\sum_{i}\left(\theta_{i} \wedge \overline{\theta_{i}}\right) \otimes a_{i},
$$

where $a_{i}, b_{i j} \in \operatorname{End}\left(E_{y}\right)$ with $a_{i}^{*}=-a_{i}$ and $b_{i j}^{*}=-b_{i j}$.

Let

$$
\Xi:=\operatorname{Tr}(\Theta \wedge \Theta) \text {. }
$$

Then $\Xi$ is a closed $(2,2)$ form which can be expressed as

$$
\sqrt{-1}^{n} \Xi \wedge \omega^{n-2}=\operatorname{Tr}\left(-\sum_{j \geq 1} b_{0 j}^{2}+a_{0} \sum_{j \geq 1} a_{j}\right) .
$$

We recall that a Higgs field on $E$ is a holomorphic section $\Phi$ of $\operatorname{End}(E) \otimes \Omega_{M}^{1,0}$ such that $\Phi \wedge \Phi=0$. A Hermitian structure on $E$ is said to satisfy the Yang-Mills equation for $(E, \Phi)$, if the Chern connection $\nabla$ on $E$ satisfies the equation

$$
\Lambda_{\omega}(\Theta+\Phi \wedge \bar{\Phi})=0
$$

where $\Theta$ as before denotes the curvature of $\nabla$.

Let $\Phi$ be a Higgs field on $E$ equipped with a Hermitian structure that satisfies the Yang-Mills equation for $(E, \Phi)$ in (4). As before, the corresponding Chern connection will be denoted by $\nabla$ and the curvature of $\nabla$ by $\Theta$. Consider the connection $\nabla^{\Phi}=\nabla+\Phi+\Phi^{*}$ on $E$. Then the curvature $\Theta^{\Phi}$ of $\nabla^{\phi}$ is given by (see Lemmas 3.2 and 3.4 of [11] for instance)

$$
\Theta_{\Phi}=\Theta+\Phi \wedge \Phi^{*}+\nabla^{(1,0)} \Phi+\nabla^{(0,1)}\left(\Phi^{*}\right) .
$$

We will denote $\nabla^{1,0} \Phi$ and $\nabla^{0,1}\left(\Phi^{*}\right)$ by $\alpha$ and $\beta$ respectively. Let

$$
\Xi^{\Phi}:=\operatorname{Tr}\left(\Theta^{\Phi} \wedge \Theta^{\Phi}\right)
$$

We note that $\Xi^{\Phi}$ is cohomologous to the discriminant of the bundle $E$, that is, the characteristic class represented by the trace of the square of its curvature, and hence the cohomology class of $\Xi_{\Phi}$ vanishes. But

$$
\Xi_{\Phi}=\Xi+\operatorname{Tr}\left(\Phi \wedge \Phi^{*} \wedge \Phi \wedge \Phi^{*}\right)+2 \cdot \operatorname{Tr}(\alpha \wedge \beta)
$$

and the signs of all three terms on the right agree with each other.

But, $\sum_{j} a_{j}=\Lambda \Theta$; hence $\sum_{j} a_{j}+\Phi \wedge \Phi^{*}=\Lambda \Theta_{\Phi}=0$. Consequently,

$$
i^{n} \Xi_{\Phi} \wedge \omega^{n-2}=\operatorname{Tr}\left(-\sum b_{i}^{2}-a_{0}^{2}\right) \text { PLUS non-negative terms. }
$$


Since

$$
\int_{M} i^{n} \Xi \wedge \omega^{n-2}=\int_{M} i^{n} \Xi_{\Phi} \wedge \omega^{n-2}=0,
$$

and $\operatorname{Tr}\left(-a_{0}^{2}\right)$ is a positive definite form on $\mathfrak{u}(E)$ (the space of element $A$ of $\operatorname{End}(E)$ such that $A^{*}=-A$ ), it follows that each $b_{i}$ is zero as is $a_{0}$.

We now have now the following analog of [14, Proposition 4.2].

Proposition 3.1. Let $\Pi: M \longrightarrow X$ be a positive principal elliptic fibration with $n=\operatorname{dim} M \geq 3$. Equip $M$ with a preferred Hermitian metric. Let $(E, \Phi)$ be a Higgs bundle with a Hermitian structure that satisfies (4). Higgs field $\Phi$ and let $\Theta_{\Phi} \in \Lambda^{1,1}(M) \otimes \operatorname{End}(E)$ be the associated endomorphism-valued (1,1)-form in (5). Then

$$
\Theta_{\Phi}(v,-)=0
$$

for any vertical tangent vector $v \in T_{v} M$.

Proposition 3.1 furnishes, as in [14, Theorem 6.1], the following. Since the proof is a replica of [14, Theorem 6.1], once Proposition 3.1 is in place, we omit it.

Theorem 3.2. Let $\Pi: M \longrightarrow X$ be a positive principal elliptic fibration with $n=\operatorname{dim} M \geq 3$. Equip $M$ with a preferred Hermitian metric. Let $E$ be a holomorphic vector bundle on $M$ admitting a Higgs field such that the resulting Higgs bundle is stable. Then $E \cong L \otimes \Pi^{*} B_{0}$, where $L$ is a holomorphic line bundle on $M$, and $B_{0}$ is a holomorphic vector bundle on $X$.

\section{Higgs field is a pullback}

Consider the set-up of Theorem 3.2. Let $T$ denote the elliptic curve that is acting on $M$. Take $E$ as in Theorem 3.2. Since

$$
E \cong L \otimes \Pi^{*} B_{0},
$$

we have

$$
\operatorname{End}(E) \cong \Pi^{*} \operatorname{End}\left(B_{0}\right) .
$$

Fix an isomorphism of $\operatorname{End}(E)$ with $\Pi^{*} \operatorname{End}\left(B_{0}\right)$.

The group $T$ has a tautological action on the pullback $\Pi^{*} \operatorname{End}\left(B_{0}\right)$. Using the isomorphism of $\operatorname{End}(E)$ with $\Pi^{*} \operatorname{End}\left(B_{0}\right)$, this action of $T$ on $\Pi^{*} \operatorname{End}\left(B_{0}\right)$ produces an action of $T$ on $\operatorname{End}(E)$. On the other hand, the action of $T$ on $M$ has a tautological lift to an action of $T$ on the cotangent bundle $\Omega_{M}^{1}$. Combining the actions of $T$ on $\operatorname{End}(E)$ and $\Omega_{M}^{1}$, we get an action of $T$ on $H^{0}\left(M, \operatorname{End}(E) \otimes \Omega_{M}^{1}\right)$.

Since the group $T$ is compact, it does not have any nontrivial holomorphic homomorphism to GL $(m, \mathbb{C})$ for any $m \geq 1$. Hence the action of $T$ on $H^{0}\left(M, \operatorname{End}(E) \otimes \Omega_{M}^{1}\right)$ is trivial. In particular, every Higgs field on $E$ is fixed by the above action of $T$ on $H^{0}\left(M, \operatorname{End}(E) \otimes \Omega_{M}^{1}\right)$.

Let

$$
\eta \in H^{0}(M, T M)
$$

be the holomorphic vector field on $M$ given by a nonzero element of Lie( $T)$ using the action of $T$ on $M$. So $\eta$ generates the line subbundle $T_{v}$ in (3).

Take a Higgs field $\Phi$ on $E$ such that the Higgs bundle $(E, \Phi)$ is stable. Since $\Phi$ is a holomorphic section of $\operatorname{End}(E) \otimes \Omega_{M}^{1}$, it follows that the contraction of $\Phi$ by the vector field $\eta$ in (6) is a holomorphic section

$$
i_{\eta} \Phi \in H^{0}(M, \operatorname{End}(E)) .
$$

Lemma 4.1. The section $i_{\eta} \Phi$ in (7) vanishes identically. 
Proof. Since $\Phi \wedge \Phi=0$, it follows immediately $\Phi$ commutes with $i_{\eta} \Phi$. (We recall that the condition $\Phi \wedge \Phi=0$ means that for any point $y \in M$, and any $v, w \in T_{y} M$, the two elements $i_{v} \Phi(y)$ and $i_{w} \Phi(y)$ of End $\left(E_{y}\right)$ commute.) Now, the stability condition of the Higgs bundle $(E, \Phi)$ implies that there is a complex number $\lambda$ such that

$$
i_{\eta} \Phi=\lambda \cdot \operatorname{Id}_{E}
$$

To prove the lemma it suffices to show that $\lambda=0$.

Consider the holomorphic 1 -form

$$
\operatorname{trace}(\Phi) \in H^{0}\left(M, \Omega_{M}^{1}\right)
$$

Evidently, we have

$$
i_{\eta}(\operatorname{trace}(\Phi))=\operatorname{trace}\left(i_{\eta} \Phi\right)=n \cdot \lambda,
$$

where $n=\operatorname{dim}_{\mathbb{C}} M$.

On the other hand, all holomorphic 1-forms on $M$ are pulled back from $X$. Indeed, this follows from the positivity of the principal elliptic fibration $\Pi: M \longrightarrow X$. Consequently, for any holomorphic 1-form $\beta$ on $M$, we have

$$
i_{\eta} \beta=0 .
$$

Applying (9) to trace $(\Phi)$, from (8) we conclude that $\lambda=0$.

Now consider $\Phi$ as a holomorphic section of $\operatorname{End}\left(\Pi^{*} B_{0}\right) \otimes \Omega_{M}^{1}$ using the chosen isomorphism of $\operatorname{End}(E)$ with $\Pi^{*} \operatorname{End}\left(B_{0}\right)=\Pi^{*} \operatorname{End}\left(B_{0}\right)$. Since $\Phi$ is fixed by the action of $T$, from Lemma 4.1 it follows immediately that the section

$$
\Phi \in H^{0}\left(M, \operatorname{End}\left(\Pi^{*} B_{0}\right) \otimes \Omega_{M}^{1}\right)=H^{0}\left(M,\left(\Pi^{*} \operatorname{End}\left(B_{0}\right)\right) \otimes \Omega_{M}^{1}\right)
$$

descends to $X$, meaning there is a section

$$
\Phi_{X} \in H^{0}\left(X, \operatorname{End}\left(B_{0}\right) \otimes \Omega_{X}^{1}\right)
$$

such that

$$
\Pi^{*} \Phi_{X}=\Phi .
$$

Since $\Phi \wedge \Phi=0$, and $\Pi$ is dominant, it follows immediately that $\Phi_{X} \wedge \Phi_{X}=0$. In other words, $\left(B_{0}, \Phi_{X}\right)$ is a Higgs bundle on $X$. It is straight-forward to check that the Higgs bundle $\left(B_{0}, \Phi_{X}\right)$ is stable with respect to $\omega_{X}$. Indeed, if a subsheaf $F$ of $B_{0}$ violates the stability condition for $\left(B_{0}, \Phi_{X}\right)$, then the subsheaf $L \otimes \Pi^{*} F$ of $L \otimes \Pi^{*} B_{0}=E$ violates the stability condition for $(E, \Phi)$.

Consequently, we have following generalization of Theorem 3.2:

Theorem 4.2. Let $\Pi: M \longrightarrow X$ be a positive principal elliptic fibration with $n=\operatorname{dim} M \geq 3$. Equip $M$ with a preferred Hermitian metric. Let $(E, \Phi)$ be a stable Higgs bundle on $M$. Then there is stable Higgs bundle $\left(B_{0}, \Phi_{X}\right)$ on $X$ and a holomorphic line bundle $L$ over $M$ such that the Higgs bundle $\left(L \otimes \Pi^{*} B_{0}, \Pi^{*} \Phi_{X}\right)$ is isomorphic to $(E, \Phi)$.

Note that Theorem 4.2 justifies the definition of a Higgs bundle on a quasi-regular Sasakian manifold adopted in [1].

Acknowledgement: The first and second authors acknowledge the support of their respective J. C. Bose Fellowships. The third author is partially supported by the Russian Academic Excellence Project '5-100'.

\section{References}

[1] I. Biswas and M. Mj, Higgs bundles on Sasakian manifolds, (2018), issue 11, 3490-3506. 
[2] S. K. Donaldson, Infinite determinants, stable bundles and curvature, Duke Math. Jour. 54 (1987), 231-247.

[3] S. Dragomir and L. Ornea, Locally conformally Kähler geometry, Progress in Mathematics, 155, Birkhäuser, Boston, MA, 1998.

[4] P. Gauduchon, La 1-forme de torsion d'une variete hermitienne compacte, Math. Ann. 267 (1984), 495-518.

[5] N. J. Hitchin, The self-duality equations on a Riemann surface, Proc. London Math. Soc. 55 (1987), 59-126.

[6] T. Höfer, Remarks on torus principal bundles, Jour . Math. Kyoto Univ. 33 (1993), 227-259.

[7] J. Li and S.-T. Yau, Hermitian-Yang-Mills connection on non-Kähler manifolds, Mathematical aspects of string theory (San Diego, Calif., 1986), 560-573, Adv. Ser. Math. Phys., 1, World Sci. Publishing, Singapore, 1987.

[8] M. Lübke and A. Teleman, The Kobayashi-Hitchin correspondence, World Scientific, Singapore, 1995.

[9] M. Lübke and A. Teleman, The universal Kobayashi-Hitchin correspondence on Hermitian manifolds, Memoirs Amer. Math. Soc. 183, No. 863, (2006).

[10] L. Ornea and M. Verbitsky, Immersion theorem for Vaisman manifolds, Math. Ann. 332 (2005), 121-143.

[11] C. T. Simpson, Constructing variations of Hodge structure using Yang-Mills theory and applications to uniformization, Jour. Amer. Math. Soc. 1 (1988), 867-918.

[12] C. T. Simpson, Higgs bundles and local systems, Inst. Hautes Études Sci. Publ. Math. 75 (1992), 5-95.

[13] K. Uhlenbeck and S.-T. Yau, On the existence of Hermitian-Yang-Mills connections in stable vector bundles, Comm. Pure Appl. Math. 39 (1986), 257-293.

[14] M. Verbitsky, Stable bundles on positive principal elliptic fibrations, Math. Res. Lett. 12 (2005), 251-264. 